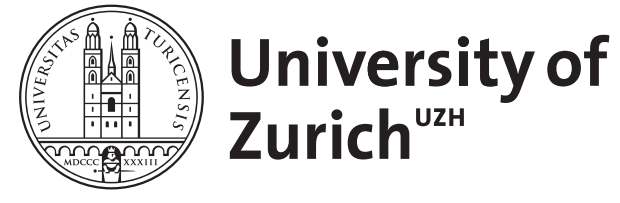

\title{
In-plane motions of the stapes in human ears
}

\author{
Lauxmann, M ; Eiber, A ; Heckeler, C ; Ihrle, S ; Chatzimichalis, M ; Huber, A ; Sim, J H
}

\begin{abstract}
The piston-like (translation normal to the footplate) and rocking-like (rotation along the long and short axes of the footplate) are generally accepted as motion components of the human stapes. It has been of issue whether in-plane motions, i.e., transversal movements of the footplate in the oval window, are comparable to these motion components. In order to quantify the in-plane motions the motion at nine points on the medial footplate was measured in five temporal bones with the cochlea drained using a three-dimensional (3D) laser Doppler vibrometer. It was found that the stapes shows in-plane movements up to $19.1 \pm 8.7 \%$ of the piston-like motion. By considering possible methodological errors, i.e., the effects of the applied reflective glass beads and of alignment of the 3D laser Doppler system, such value was reduced to be about $7.4 \pm 3.1 \%$. Further, the in-plane motions became minimal ( $4.2 \pm 1.4 \%$ of the piston-like motion) in another plane, which was anatomically within the footplate. That plane was shifted to the lateral direction by $118 \mathrm{~m}$, which was near the middle of the footplate, and rotated by $4.7^{\circ}$ with respect to the medial footplate plane.
\end{abstract}

DOI: https://doi.org/10.1121/1.4756925

Posted at the Zurich Open Repository and Archive, University of Zurich ZORA URL: https://doi.org/10.5167/uzh-69197

Journal Article

Published Version

Originally published at:

Lauxmann, M; Eiber, A; Heckeler, C; Ihrle, S; Chatzimichalis, M; Huber, A; Sim, J H (2012). In-plane motions of the stapes in human ears. Journal of the Acoustical Society of America, 132(5):3280-91.

DOI: https://doi.org/10.1121/1.4756925 


\title{
In-plane motions of the stapes in human ears
}

\author{
M. Lauxmann, a) A. Eiber, C. Heckeler, and S. Ihrle \\ Institute of Engineering and Computational Mechanics, University of Stuttgart, Pfaffenwaldring 9 , \\ 70569 Stuttgart, Germany \\ M. Chatzimichalis, A. Huber, and J. H. Sim \\ Department of Otorhinolaryngology, Head and Neck Surgery, University Hospital Zurich, \\ Frauenklinkstrae 24, 8091 Zurich, Switzerland
}

(Received 10 May 2012; revised 8 September 2012; accepted 13 September 2012)

\begin{abstract}
The piston-like (translation normal to the footplate) and rocking-like (rotation along the long and short axes of the footplate) are generally accepted as motion components of the human stapes. It has been of issue whether in-plane motions, i.e., transversal movements of the footplate in the oval window, are comparable to these motion components. In order to quantify the in-plane motions the motion at nine points on the medial footplate was measured in five temporal bones with the cochlea drained using a three-dimensional (3D) laser Doppler vibrometer. It was found that the stapes shows in-plane movements up to $19.1 \pm 8.7 \%$ of the piston-like motion. By considering possible methodological errors, i.e., the effects of the applied reflective glass beads and of alignment of the 3D laser Doppler system, such value was reduced to be about $7.4 \pm 3.1 \%$. Further, the in-plane motions became minimal ( $\approx 4.2 \pm 1.4 \%$ of the piston-like motion) in another plane, which was anatomically within the footplate. That plane was shifted to the lateral direction by $118 \mu \mathrm{m}$, which was near the middle of the footplate, and rotated by $4.7^{\circ}$ with respect to the medial footplate plane. (C) 2012 Acoustical Society of America. [http://dx.doi.org/10.1121/1.4756925]
\end{abstract}

PACS number(s): 43.64.Ha, 43.64.Bt [BLM]

Pages: $3280-3291$

\section{INTRODUCTION}

Measuring motions of the middle-ear structures is one of the most widely-used techniques for experimental investigations of middle-ear mechanics. Many previous investigations have focused on the physiological motion of the stapes as an output of sound transmission through the middle ear and a linkage between the middle and inner ears. A comparison of middle-ear transfer functions across several species was performed by Ruggero and Temchin (2003), and vibration patterns of the stapes in the frequency domain differed with species. In human subjects, while vibration amplitude of the stapes were different depending on experimental methods and temporal bone (TB) conditions (Rosowski et al., 2004; Chien et al., 2006), the first resonance of the middle-ear motion was similar. Silman and Silverman (1991) reported that the first resonance of the middle-ear is around 0.8 to $1.2 \mathrm{kHz}$, and measurements carried out by Wada et al. (1998) on 275 ears from live human subjects showed the mean resonance frequency of $1.17 \pm 0.27 \mathrm{kHz}$ for air conduction.

Though it has been assumed that the stapes moves mainly in a piston-like manner, spatial modes of the stapes vibration have been reported also. The stapes motion in human TBs was described by von Békésy (1960) under moderate amplitudes of stimulation as rotating about the vertical (i.e., in the superior-inferior direction) axis running through a point near the posterior edge of the footplate. This observation corresponded with the anatomical feature of the annular

\footnotetext{
a) Author to whom correspondence should be addressed. Electronic mail: Michael.Lauxmann@itm.uni-stuttgart.de
}

ligament, which is wide and thin in the anterior portion and narrow and thick in the posterior portion. Kirikae (1960) performed similar experiments and concluded that the vibration pattern of the stapes footplate is a combination of three movements: Piston-like motion, hinged motion around a posterior axis, and rotation around the long axis. Both von Békésy and Kirikae used TBs with drained cochleae in their studies. Gundersen (1972) showed that a piston-like motion pattern is preserved up to $2 \mathrm{kHz}$ but at higher frequencies rocking-like motions become apparent. However, his measurement technique of electromagnetically picking-up the stapes vibrations may have influenced the vibrational modes due to the added load by the pick-up needle contacting the stapes. Recent developments in measurement techniques and methods have confirmed spatial modes of the stapedial vibrations. Using a video measuring system, Gyo et al. (1987) observed predominant piston-like movements only at low frequencies and complex movements at higher frequencies. Other measurements with laser Doppler vibrometer (LDV) systems have also shown spatial modes of the stapes vibration in humans and animals, especially at high frequencies such as reported by Decraemer and Khanna (1999), Voss et al. (2000), Hato et al. (2003), Decraemer et al. (2007), Ravicz et al. (2008), and Sim et al. (2010a). Therefore, it has been generally accepted that at high frequencies the stapes exhibits not only piston-like but also complex spatial motions.

To describe the spatial motions of the stapes as a rigid body, three translational and three rotational degrees of freedom are required. In our previous works (Sim et al., 2010a, 2010b) and in the work by Hato et al. (2003), for simplicity of the measurement, only the piston-like motion and two 
rocking-like motions, which are rotations along the long and short axes of the footplate, were considered as primary motion components (e.g., in a human and a guinea pig). An assumption made in those works was that the annular ligament suppressed the in-plane motions of the stapes footplate. However, Decraemer et al. (2007) found non-negligible inplane motions in the gerbil stapes, with the in-plane motions of the footplate plane in a similar range to the piston-like component. As for humans, no measurements showing the full six-degrees-of-freedom stapes motion patterns have been published. Due to the surface shape of the stapes footplate, in-plane motions are presumed to have little effect on the fluid-flux in the cochlea and thus little effect on hearing. However, they may affect the calculation of the assumed primary motion components (i.e., the piston-like motion and the two rocking-like motions).

This study aimed to identify the six-degrees-of-freedom and the precise spatial vibration modes of the human stapes by measuring spatial motion of selected points on the footplate with a three-dimensional (3D) LDV system. For the best estimation of the stapes orientation in the measurement frame, footplate motions were captured from the vestibuli side with the cochlea drained. The in-plane motion components were compared to the piston- and rocking-like components. Furthermore, how uncertainties in the relative alignment between the laser and the stapes and uncertainties in the position of the measurement points on the stapes footplate influenced the reconstruction of the stapes motion and thus the magnitude of the in-plane motion components was examined.

\section{MATERIAL AND METHODS}

\section{A. Temporal bone preparation and experimental setup}

Five fresh TBs were harvested from the right side of human cadavers within $24 \mathrm{~h}$ after death and placed in Thiomersal solution at $4{ }^{\circ} \mathrm{C}$. All measurements were performed within one week of explantation. After removal of all connective tissue and muscles, the external ear canal was drilled down to $2 \mathrm{~mm}$ from the tympanic annulus. An artificial ear canal (AEC) approximately $0.5 \mathrm{ml}$ in volume was attached to the bony rim of the former external ear canal. While the middle-ear structures, including ligaments, tendons, and the tympanic membrane, were kept intact, most parts of the cochlea were removed to enable visualization of the vestibular side of the stapes footplate from the vestibuli side (i.e., the medial side of the footplate). The medial surface of the footplate was completely coated with retro-reflective beads (50 $\mu \mathrm{m}$ diameter) to improve reflectivity of the laser beams of the 3D LDV system. To prevent drying out of the specimen, pieces of paper towel soaked in water were placed all around the specimen before it was enveloped in plastic wrap.

The TBs were mounted on a custom-made holder, and alignment of the stapes footplate was performed with a surgical microscope. The TB holder was placed in a test rig such that the long axis ( $x$-axis) and short axis (z-axis) of the footplate were oriented along the axes of the test rig frame. Figure 1 gives an overview of the measurement setup with the stapes coordinate system. Further, a 3D LDV system (CLV-3000, CLV-3D, Polytec, Germany) was mounted on

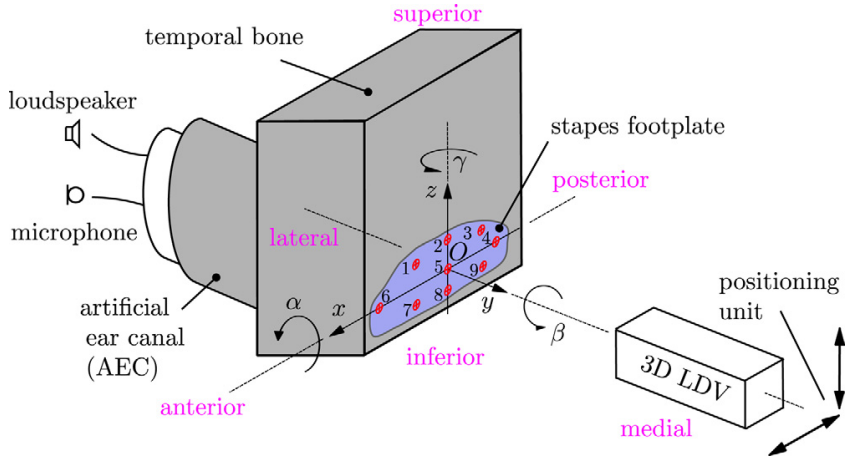

FIG. 1. (Color online) Physiological motions of the stapes were measured from the medial side at nine points of the footplate with a 3D LDV. The figure displays the measurement coordinate system with its origin in the centroid of the footplate and its six-degrees-of-freedom. The optical axis of the 3D LDV was perpendicular to the approximated plane of the stapes footplate. A loudspeaker and a microphone were placed in an AEC.

the test rig such that the optical axis of the laser system was perpendicular to the visually approximated plane of the stapes footplate's surface. Using electrically driven translation stages with internal displacement encoders, the location of the 3D LDV could be positioned to any point on the stapes footplate, with an accuracy of $1 \mu \mathrm{m}$ in both vertical and horizontal directions. A USB microscopic camera (Digimicro 1.3 , DNT, Germany) was coupled onto the optical axis of the 3D laser by means of a reflective prism to enable visual observation of the laser spot position on the medial surface of the stapes footplate.

\section{B. Measurement}

Four sets of harmonic multi-frequency signal ( 0.1 to $1.5,1.6$ to $4.5,4.75$ to 7 , and 7.5 to $10 \mathrm{kHz}$ ) were delivered in sequence to a loud speaker (ER-2, Etymotic Research, Elk Grove Village, IL) placed in the AEC. The frequency steps were $100 \mathrm{~Hz}$ up to $2 \mathrm{kHz}, 250 \mathrm{~Hz}$ between 2 and $5 \mathrm{kHz}$, and $500 \mathrm{~Hz}$ above $5 \mathrm{kHz}$. Thus, 15 frequencies were included in each of the first 2 sets, and 6 frequencies in each of the other 2 sets, resulting in the total 42 frequencies in the frequency range of $0.1 \mathrm{~Hz}$ to $10 \mathrm{kHz}$. The sound pressure level (SPL) in the AEC was monitored by a microphone (ER-14C, Etymotic Research, Elk Grove Village, IL). AEC pressure levels of greater than $90 \mathrm{~dB}$ SPL up to $6 \mathrm{kHz}$ and $75 \mathrm{~dB}$ SPL above $6 \mathrm{kHz}$ were obtained.

Motion of the stapes in response to each of the four sets of harmonic multi-frequency signal was measured by the 3D LDV system, sequentially at nine points on the stapes footplate, including the center, four edge points (anterior, posterior, superior, and inferior edges), and four intermediate points between the edge points and the center of the stapes footplate (see Fig. 1). First the laser spot was positioned on the visually estimated centroid of the footplate, which was considered as origin. Then, the coordinates of each measurement point on the footplate were taken from the encoders. The measurement was automatically performed by a custom-made algorithm.

Measurement at each point was repeated 100 times (100 measurement blocks). Constant and linear trends were removed from the recorded velocity data using a built-in 
function detrend provided by MATLAB (The Mathworks Inc., Natick, MA), and corrupted measurement blocks showing large deviations from other data blocks were excluded. From the remaining data sets, the transfer functions between the pressure in the AEC (input) and the spatial velocities (output) were calculated as the quotient of the cross power spectral densities. The transfer functions were averaged, and spatial displacements $\boldsymbol{u}_{i}$ at the points $P_{i}$ were obtained by integration of the velocities with respect to time.

\section{3D components of stapes motion}

Rigid body motions of the stapes can be described with three translational components and three rotational components about a reference point in a reference frame. These six kinematical quantities of the stapes are called generalized coordinates of a rigid body motion. In this section, we describe how the rigid-body motion components of the spatial stapes motion were derived in a reference frame based on the medial surface of the stapes footplate.

The uneven medial surface of the stapes footplate was approximately considered as planar which is further denoted as the $K_{0}$-plane. The corresponding frame (coordinate system) was defined such that the origin $O$ was at the centroid of the approximated footplate surface, the $x_{K_{0}}$-axis was along the long axis of the footplate with the anterior direction as its positive direction, the $z_{K_{0}}$-axis running along the short axis of the footplate with the superior direction as its positive direction, and the $y_{K_{0}}$-axis completing a right-handed reference system and thus being orthogonal to the $K_{0}$-plane. The $K_{0}$-frame and the corresponding six rigid-body motion components are shown in Fig. 1.

Denoting in the $K_{0}$-frame the three translational displacements of the origin as $x_{0}, y_{0}$, and $z_{0}$, and the three rotational displacements of the stapes as $\alpha_{0}, \beta_{0}$, and $\gamma_{0}$, then the linearized $3 \mathrm{D}$ displacement components $u_{x i}, u_{y i}$, and $u_{z i}$ at a measurement point $P_{i}$ are related to the six rigid-body motion displacements of the stapes as

$$
\boldsymbol{u}_{i}=\boldsymbol{u}_{O}+\boldsymbol{\vartheta}_{K_{0}} \times \boldsymbol{r}_{O P_{i}},
$$

with

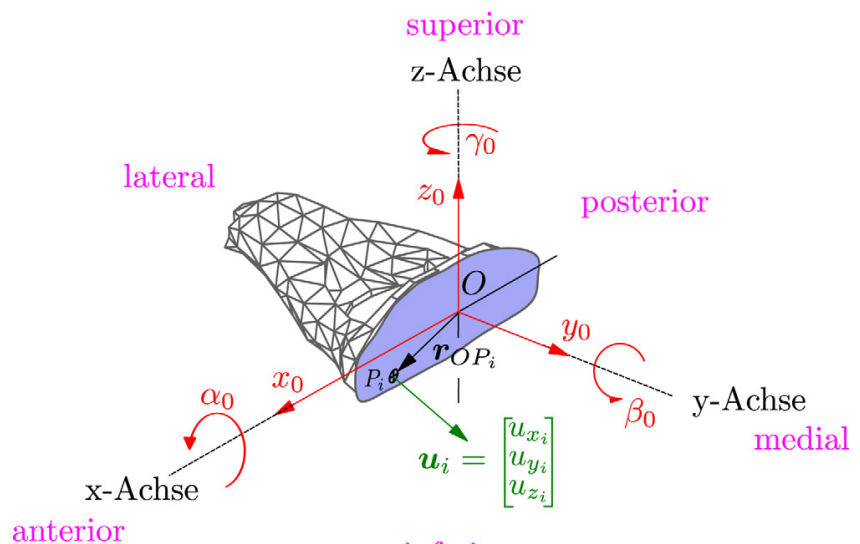

anterior

$$
\text { inferior }
$$

FIG. 2. (Color online) Description of the stapes rigid body motion with the three translational displacements of the origin as $x_{0}, y_{0}$, and $z_{0}$, and the three rotational displacements of the stapes as $\alpha_{0}, \beta_{0}$, and $\gamma_{0}$. $\boldsymbol{u}_{i}=\left[\begin{array}{l}u_{x_{i}} \\ u_{y_{i}} \\ u_{z_{i}}\end{array}\right], \boldsymbol{u}_{O}=\left[\begin{array}{l}x_{0} \\ y_{0} \\ z_{0}\end{array}\right], \boldsymbol{\vartheta}_{K_{0}}=\left[\begin{array}{l}\alpha_{0} \\ \beta_{0} \\ \gamma_{0}\end{array}\right]$ and $\boldsymbol{r}_{O P_{i}}=\left[\begin{array}{c}x_{i}^{\prime} \\ y_{i}^{\prime} \\ z_{i}^{\prime}\end{array}\right]$

where $\boldsymbol{r}_{O P_{i}}$ represents a body-fixed position vector pointing from the origin $O$ to the point $P_{i}$ in the $K_{0}$-frame as Fig. 2 illustrates.

With $\boldsymbol{q}_{0}$ denoting a vector with the six rigid-body motion components, Eq. (1) can be rewritten as

$$
\boldsymbol{u}_{i}=\boldsymbol{A}_{i} \boldsymbol{q}_{0}
$$

with

$$
\boldsymbol{A}_{i}=\left[\begin{array}{rrrrrr}
1 & 0 & 0 & 0 & z_{i}^{\prime} & -y_{i}^{\prime} \\
0 & 1 & 0 & -z_{i}^{\prime} & 0 & x_{i}^{\prime} \\
0 & 0 & 1 & y_{i}^{\prime} & -x_{i}^{\prime} & 0
\end{array}\right] \text { and } \boldsymbol{q}_{0}=\left[\begin{array}{c}
x_{0} \\
y_{0} \\
z_{0} \\
\alpha_{0} \\
\beta_{0} \\
\gamma_{0}
\end{array}\right]
$$

Combining all $n$ measurement points, this leads to

$$
\boldsymbol{u}=\boldsymbol{A} \boldsymbol{q}_{0}
$$

with $\boldsymbol{u}=\left[\boldsymbol{u}_{1}, \boldsymbol{u}_{2}, \ldots, \boldsymbol{u}_{n}\right]^{T}$ and $\boldsymbol{A}=\left[\boldsymbol{A}_{1}, \boldsymbol{A}_{2}, \ldots, \boldsymbol{A}_{n}\right]^{T}$. The vector $\boldsymbol{u}$ contains the measured displacements of the $n=9$ points and $\boldsymbol{A}$ is the matrix determined from the respective coordinates $x_{i}^{\prime}, y_{i}^{\prime}$, and $z_{i}^{\prime}$ of each of the $n$ measurement points.

Ideally, the six rigid-body motion components should be able to be derived from 3D LDV measurements at three noncollinear points on the footplate. However, due to unavoidable measurement inaccuracies, the measurements were done at nine points, resulting in an over-determined system in Eq. (3) allowing an error compensation. The method of least squares error was used to calculate the six rigid-body motion components as

$$
\boldsymbol{q}_{0}=\left(\boldsymbol{A}^{T} \boldsymbol{A}\right)^{-1} \boldsymbol{A}^{T} \boldsymbol{u} .
$$

In the experiments, sets of multi-frequency harmonic sound stimuli were used (see Sec. IIB). As the middle ear is presumed to act as a linear system with applied SPL below $120 \mathrm{~dB}$ Voss et al. (2000), the measured displacements $\boldsymbol{u}_{i}$ and the resulting rigid-body motion component vector $\boldsymbol{q}_{0}$ by harmonic stimulation can be regarded harmonic as well. According to Eq. (4), the amplitude and phase of $\boldsymbol{q}_{0}$ were then calculated separately for each of all 42 experimental frequencies that were included in the stimulus signal.

\section{Footplate-plane used for minimal in-plane motion and in-plane motion parameter}

Generally a point on an arbitrary cross-sectional plane of the stapes performs a spatial motion. The point motion at any instant can be decomposed into a motion component out of the plane and a motion component in the plane. The magnitudes of the decomposed components are dependent on the position and orientation of the plane under focus. A specific 
plane can be found in which the in-plane motions are minimal or even zero in some cases of reduced degrees of freedom such as a pure translation. In the following, this particular plane is referred to as the $K_{1}$-plane. The hypothesis was proposed that the $K_{1}$-plane is located within the stapes footplate body, since the annular ligament surrounds the stapes footplate and serves as a joint attenuating some of the six rigid body motions.

In the $K_{0}$-plane, as the position coordinate $y_{i}^{\prime}$ of a measurement point $P_{i}$ equals zero, the in-plane motions $u_{x i}$ and $u_{z i}$ depend only on the three rigid-body motion components $x_{0}, z_{0}$, and $\beta_{0}$, according to Eq. (2). Thus, the magnitudes of these three rigid-body motion components were taken as a measure of in-plane motions in the $K_{0}$-plane.

Aiming to investigate the in-plane motions in the $K_{1}$-plane, the rigid body motion of the stapes is described with respect to the corresponding stapes frame $K_{1}$, whose $x_{K_{1}}-z_{K_{1}}$ plane represents the $K_{1}$-plane. Figure 3 illustrates the stapes and the two frames $K_{0}$ and $K_{1}$ from a superior view. The $K_{1}$-frame is characterized relatively to the $K_{0}$-frame by means of a translational vector $\boldsymbol{r}_{O O_{1}}=\left[\begin{array}{lll}x_{O O_{1}} & y_{O O_{1}} & z_{O O_{1}}\end{array}\right]^{T}$ from the origin $O$ of the $K_{0}$-frame to the origin $O_{1}$ of the $K_{1}$-frame, and a rotational transformation matrix $S_{K_{0} K_{1}}$ from the $K_{0}$-frame to the $K_{1}$-frame. The rotational transformation matrix contains the three Bryant angles $\alpha_{K_{0} K_{1}}, \beta_{K_{0} K_{1}}$, and $\gamma_{K_{0} K_{1}}$, which are rotation angles of the $K_{1}$-frame with respect to $x, y$, and $z$ axes in sequence.

In the $K_{1}$-frame, the stapes motion is described in terms of the translation $\boldsymbol{u}_{O 1}=\left[\begin{array}{lll}x_{1} & y_{1} & z_{1}\end{array}\right]^{T}$ and rotation $\boldsymbol{\vartheta}_{K 1}=\left[\begin{array}{ll}\alpha_{1} & \beta_{1} \\ \gamma_{1}\end{array}\right]^{T}$ of the origin $O_{1}$. Having described the stapes motion in the $K_{1}$-frame, the in-plane motions of the $K_{1}$-plane are represented by the three rigid-body motion components $x_{1}, z_{1}$, and $\beta_{1}$. In the following, the relation between $x_{0}, z_{0}$, and $\beta_{0}$ in the $K_{0}$-frame and $x_{1}, z_{1}$, and $\beta_{1}$ in the $K_{1}$-frame is derived.

The displacement $\boldsymbol{u}_{O_{1}}$ of the origin $O_{1}$ of the $K_{1}$-frame is represented by the translation $\boldsymbol{u}_{O}$ and rotation $\boldsymbol{\vartheta}_{K 0}$ in the $K_{0}$-frame as

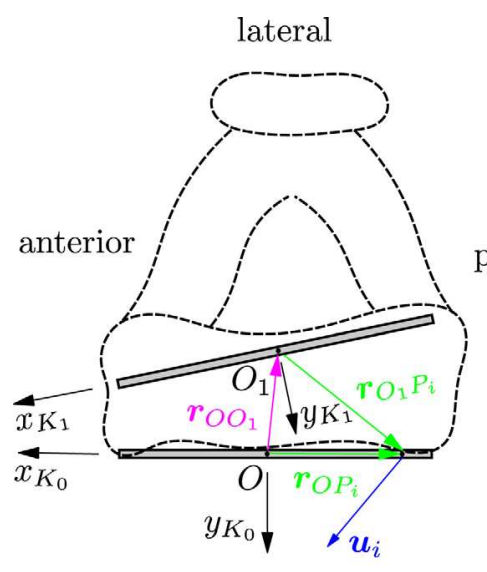

medial posterior

\section{stapes frame $K_{1}$}

measurement frame $K_{0}$ (medial surface of the footplate)
FIG. 3. (Color online) Illustration of the stapes from a superior view. The footplate thickness is scaled up for illustration purposes. Within the footplate two frames of reference $K_{0}$ and $K_{1}$ are displayed. The rigid body motion of the stapes can be described with respect to any frame of reference, e.g., with respect to $K_{0}$ or $K_{1}$. This enables calculations of the corresponding in-plane parameters in the $K_{0}$ - and $K_{1}$-plane.

$$
\boldsymbol{u}_{O 1}=\left[\begin{array}{c}
x_{1} \\
y_{1} \\
z_{1}
\end{array}\right]=\boldsymbol{S}_{K_{0} K_{1}}^{T}\left(\boldsymbol{u}_{O}+\boldsymbol{\vartheta}_{K_{0}} \times \boldsymbol{r}_{O O_{1}}\right)
$$

using Eq. (1) and the identity $\boldsymbol{S}_{K_{0} K_{1}}^{-1}=\boldsymbol{S}_{K_{0} K_{1}}^{T}$ for orthogonal matrices. The rotational components $\boldsymbol{\vartheta}_{K 0}$ are of the firstorder approximation with their small magnitudes; therefore, the transformation law can be applied for the rotational components between $K_{0}$ - and $K_{1}$-frames, and it yields

$$
\boldsymbol{\vartheta}_{K_{1}}=\left[\begin{array}{c}
\alpha_{1} \\
\beta_{1} \\
\gamma_{1}
\end{array}\right]=\boldsymbol{S}_{K_{0} K_{1}}^{T} \boldsymbol{\vartheta}_{K_{0}}
$$

Equations (5) and (6) show how the rigid body motion components in the $K_{1}$-frame can be calculated from the rigidbody motion components in the $K_{0}$-frame when the shift $\boldsymbol{r}_{O O_{1}}$ and the rotation $S_{K_{0} K_{1}}$ for transformation between the two frames are given.

Starting from the rigid-body motion components of the $K_{0}$-frame, the position and orientation of the $K_{1}$-plane was searched for such that the in-plane components $x_{1}, z_{1}$, and $\beta_{1}$ were minimal. With the coordinates of the translation vector $\boldsymbol{r}_{O O_{1}}$ and the Bryant angles of the rotational transformation matrix $\boldsymbol{S}_{K_{0} K_{1}}$ as design variables, a solution $\boldsymbol{p}^{*}=\left[x_{O O_{1}}^{*} y_{O O_{1}}^{*}\right.$ $\left.z_{O O_{1}}^{*} \alpha_{K_{0} K_{1}}^{*} \beta_{K_{0} K_{1}}^{*} \gamma_{K_{0} K_{1}}^{*}\right]$ of a minimization problem was calculated, which fulfilled the criterion

$$
\min _{p}\left(\sum_{k=1}^{s}\left|x_{1, k}\right|+\left|z_{1, k}\right|+\left|\beta_{1, k}\right|\right)
$$

where the index $k$ indicates the $k$ th frequency from the $s=42$ frequencies included in the stimulus signal. This minimization problem was solved using a built-in function fmincon in MATLAB (The Mathworks Inc., Natick, MA).

To assess magnitudes of the in-plane motion components $x_{i}, z_{i}$, and $\beta_{i}$, they were related to $y_{i}$. In the case of the $K_{0}$-frame, $y_{0}$ is commonly referred to as a "piston-like motion." The in-plane motion parameters were defined with respect to the general frame $K_{j}$

$$
T_{x j}=\frac{\left|x_{j}\right|}{\left|y_{j}\right|}, T_{z j}=\frac{\left|z_{j}\right|}{\left|y_{j}\right|} \text { and } T_{\beta_{j}}=\frac{\left|\beta_{j}\right| 0.7 \mathrm{~mm}}{\left|y_{j}\right|} .
$$

Regarding the length of the long (about $1.5 \mathrm{~mm}$ ) and short (about $0.7 \mathrm{~mm}$ ) axes of the footplate, the angular rotation $\beta_{j}$ was multiplied with a distance of $0.7 \mathrm{~mm}$ to get a corresponding translational in-plane motion component. In Eq. (8), $j=0$ and $j=1$ refer to the in-plane parameters of the $K_{0}$-plane and the $K_{1}$-plane, respectively. These parameters illustrate magnitude ratios of the in-plane motion components in the plane to the motion component normal to the plane.

\section{IIlustrative example}

The following fictive example illustrates the idea of introducing the $K_{0^{-}}$and $K_{1}$-planes and the in-plane parameters. For simplicity, the stapes was assumed to exhibit 
only two non-zero rigid-body motion components in the $K_{0}$-frame: A translational motion along the $y_{K_{0}}$-axis and a small translational motion of the origin along the $x_{K_{0}}$-axis. Both motion components were assumed to be in phase. Since the stapes exhibits no rotation, all points of the body move in the same manner. Hence, the resultant displacement vector $\boldsymbol{u}_{i}$ of all points of the body has the same magnitude and direction. Figure 4(A) illustrates the displacement components $u_{x i}$ and $u_{z i}$, and the resultant displacement vector $\boldsymbol{u}_{i}$ of three points of the $K_{0}$-plane. Obviously, the points in the $K_{0}$-plane have an in-plane motion component $u_{x i}$. In Fig. 4(B), an inclined plane perpendicular to $u_{i}$, denoted as the $K_{1}$-plane, is shown, in which the motion of all points have no in-plane components. The inclination angle $\varphi$ between the $y_{K_{0}}$-axis and the $y_{K_{1}}$-axis can be calculated analytically using the tangent of $u_{x_{i}}$ by $u_{y_{i}}$. In the case that $u_{x_{i}}$ is a tenth of $u_{y_{i}}$, the angle $\varphi$ yields to $5.7^{\circ}$.In this case, $T_{x_{0}}$ equals $0.1(10 \%)$ and $T_{x_{1}}$ equals 0 . The two other in-plane parameters $T_{z_{i}}$ and $T_{\beta_{i}}$ are obviously zero for both $K_{0^{-}}$and $K_{1}$-planes.

This example demonstrates that in-plane motions can only be interpreted with respect to a specific plane. Under a certain rigid body motion of the stapes, magnitudes of inplane motions are different depending on the cross-sectional plane under consideration.

\section{E. Error analysis}

In this section, we provide a phenomenological discussion on errors induced by the methods used in this study. First, the uncertainties in the position of the measurement points due to the presence of a retro-reflective glass bead layer on the footplate are discussed. Next, we discuss how uncertainties in the alignment of the $3 \mathrm{D} \mathrm{LDV}$ with respect to the stapes affect the determined in-plane motions. The mathematical description of the resulting errors in the in-plane parameters caused by these uncertainties is appended (see the Appendix).

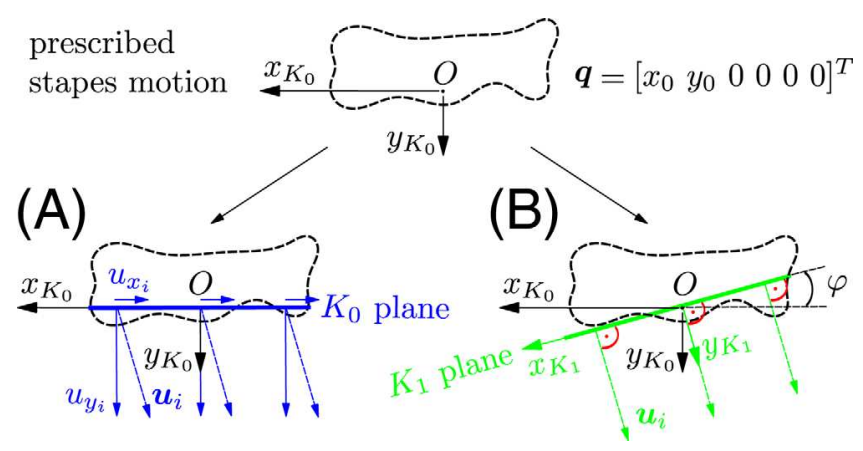

FIG. 4. (Color online) The stapes exhibits a rigid body motion prescribed in $K_{0}$ by the generalized coordinates $\boldsymbol{q}_{0}$, whereby $x_{0}$ and $y_{0}$ are in phase. Since the stapes exhibits no rotation, all points of the body move in the same manner. Hence, the resultant displacement vector $\boldsymbol{u}_{i}$ is pointing along the same direction at all points. (A) and (B) illustrate the resulting displacement vectors of three arbitrary points in the $K_{0^{-}}$and $K_{1}$-planes, respectively. The points of the $K_{0}$-plane (A) obviously have an in-plane motion component $u_{x_{i}}$, whereas the points of the inclined $K_{1}$-plane (B) do not because $\boldsymbol{u}_{i}$ stands perpendicular to the plane. The angle $\varphi$ can be calculated using the tangent of $u_{x_{i}}$ divided by $u_{y_{i}}$.

\section{Uncertainties in the measurement positions caused by the glass beads}

The medial surface of the footplate was completely covered with retro-reflective glass beads of $50 \mu \mathrm{m}$ diameter in order to increase the reflectivity of the laser beam. Several layers were required to ensure complete coverage of the entire footplate. Thereby, it is presumed that the actual measurement frame (denoted as the $K_{O^{\prime}}$-frame in this study) was shifted toward the medial direction (i.e., positive $y_{K_{0}}$-direction) by $b \approx 50$ to $300 \mu \mathrm{m}$ with respect to the $K_{0}$-frame, as shown in Fig. 5. The unevenness of the actual measurement surface was not considered here.

In order to illustrate the consequences of the shift between the $K_{0^{-}}$and $K_{0^{\prime}}$-planes exemplarily, Fig. 5 depicts the displacements at three measurement points in the $K_{0^{\prime}}$-plane for a specific case where motion of the stapes has only a rotation around the $z_{K_{0}}$-axis (short axis of the footplate) by an angle $\gamma_{0}$. While points on the $K_{0}$-plane do not have in-plane motions, points on the $K_{0^{\prime}}$-plane move out of the $K_{0^{\prime}}$-plane by $u_{y_{i}^{\prime}}$ and in the plane by $u_{x_{i}^{\prime}}$, whereas $u_{x_{i}}=b \gamma_{0}$. Measurements by the 3D LDV picked up the displacements $u_{y_{i}^{\prime}}$ and $u_{x_{i}^{\prime}}$ on the $K_{0^{\prime}}$-plane instead of $u_{y_{i}}$ and $u_{x_{i}}$ on the $K_{0}$-plane. Therefore, if the offset $b$ is not considered in reconstruction of the stapes motion (i.e., $b$ set to zero), the $K_{0}$-frame is treated as the actual measurement frame instead of the $K_{0^{\prime}}$-frame, resulting in errors on the estimation of the displacements $u_{y_{i}}$ and $u_{x_{i}}$. As a result, the reconstruction of motion indicates that there are in-plane motions in the $K_{0}$-plane, even when they do not exist.

To assess the consequences of the glass beads on the inplane motion parameter, a physiological stapes motion at $1000 \mathrm{~Hz}$ and $94 \mathrm{~dB}$ SPL with three-degrees-of-freedom, without any in-plane motions in the $K_{0}$-plane, was taken as an example. Then Eqs. (14) and (15) deliver for $b$ from 50 to $300 \mu \mathrm{m}$ incorrect in-plane parameters: $T_{x}$ from $0.7 \%$ to $4.4 \%$ and $T_{z}$ from $0.9 \%$ to $5.5 \%$ instead of $T_{x}=T_{z}=0 . T_{\beta}$ was not affected in this case.

\section{Uncertainties in the laser alignment}

To see the effect of misalignment of the laser on the obtained in-plane motion, it was assumed that the $K_{0^{\prime}}$-plane

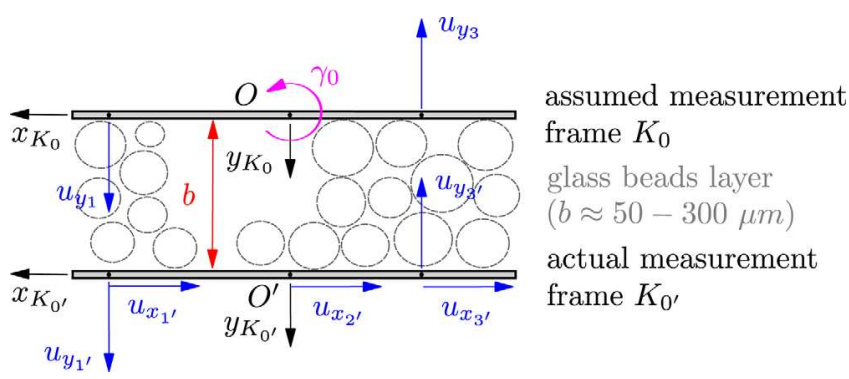

FIG. 5. (Color online) The displacements at three measurement points in the $K_{O^{\prime}}$-plane for the specific case where the stapes is rotating around the $z_{K_{O}}$ axis by the angle $\gamma_{0}$ are depicted. Due to the retro-reflective glass beads the actual measurement plane $K_{O^{\prime}}$ is possibly shifted toward the medial direction with respect to the $K_{0}$-plane by $b \approx 50$ to $300 \mu \mathrm{m}$. Without the offset $b$ considered, the recorded displacements $u_{x_{i}}$ and $u_{y_{i}}$ are incorrectly assigned to the points in the $K_{0}$-plane. Hence, it seems that there are in-plane motions $\bar{u}_{x_{i}}$ in the $K_{0}$-plane, although they are not physically present in that plane. 
coincided with the $K_{0}$-plane, i.e., the glass beads layer was not present. Ideally, the optical axis ( $y_{L}$-axis) of the 3D LDV is perpendicular to the $K_{0^{\prime}}$-plane. However, due to inevitable uncertainties in the alignment of the stapes to the TB holder, as well as in the alignment of the holder and the 3D LDV to the test rig, the actual optical axis $\left(K_{L^{\prime}}\right.$-frame) was possibly rotated against the $K_{0^{\prime}}$-plane by the spatial angle $\varphi$ between the $y_{K_{0^{\prime}}}$-axis and the $y_{K_{L^{\prime}}}$-axis, as shown in Fig. 6.

In order to get an idea about realistic error bounds on the misalignment of the 3D LDV system, accuracy of the visual alignment was assessed. In the literature, the misalignment between the LDV beam and the vertical axes of the stapes footplate is often estimated to be within $\pm 5^{\circ}$ (e.g., Hato et al., 2003). For the purpose of determining the realistic bounds of our setups, a scanning laser Doppler vibrometer (SLDV) system (OFV-3001, Polytec, Germany) was mounted in the test rig, and the deviation of the actual alignment to the assumed alignment was estimated using a method that we have reported previously (Sim et al., 2010b, 2010a). The laser beam of the SLDV system had an azimuth angle of $34.5^{\circ}$ and an elevation angle of $3.7^{\circ}$ from the normal position of the assumed stapes footplate. The azimuth and elevation angles were measured with a lever meter and a protractor. Four pieces of small wires were attached and glued to the peripheral bones near the oval window using instant glue gels (cyanoacrylate based fast-acting adhesives in a jelly-like state), and their outlines were used as references to obtain the relation between the SLDV measurement frame and the footplate-fixed frame (i.e., $\mathrm{K}_{0}$-frame). The coordinates of the outline of reference wires in the SLDV measurement frame were recorded by the PSV V8.5 software (Polytec GmbH, Germany) of the SDLV system. After the motion measurements, the TBs with the reference wires were scanned using a micro-CT machine (CT40, Scanco Medical AG, Switzerland), from which the 3D features and relative positions of the stapes and reference wires were obtained and registered into the footplate-fixed frame. The relation between the SLDV measurement frame and the footplate-fixed frame calculated, the measured coordinates of the outline in the SLDV measurement frame and real out-

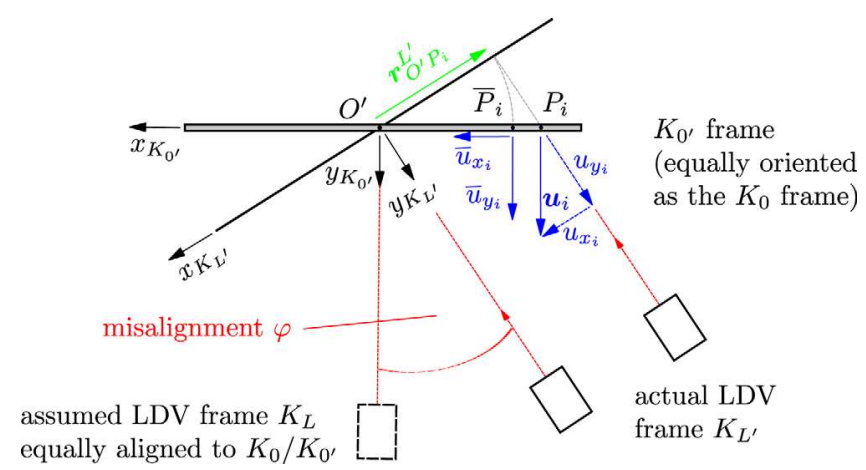

FIG. 6. (Color online) The 3D LDV frame $K_{L}$ was supposed to coincide with the measurement frame $K_{0^{\prime}}$. Due to inevitable uncertainties in the alignment, the actual LDV frame $K_{L^{\prime}}$ may be rotated against the assumed frame $K_{0^{\prime}}$. Thus, the displacements $u_{x_{i}}$ and $u_{y_{i}}$ are measured at the point $P_{i}$ with respect to the laser frame but they are incorrectly assigned to the point $\bar{P}_{i}$ and incorrectly interpreted as the vectors $\bar{u}_{x_{i}}$ and $\bar{u}_{y_{i}}$ with respect to the $K_{0^{\prime}}$ frame. lines of the reference wires obtained from micro-CT imaging. Details of these methods have been described in our previous work (Sim et al., 2012). Using this method, we determined a mean deviation of $7.5^{\circ}$ with a standard deviation of $3.4^{\circ}$ from all five TBs. This provided a reference value of the accuracy of the visual alignment of the 3D LDV with respect to the stapes.

The misalignment of the laser causes an error in the positions of the measurement points $P_{i}$ as well as the measured displacements $\boldsymbol{u}_{i}$ at that point. The position error stems from the fact that the location of the measurement point $\boldsymbol{r}_{O P_{i}}$ was determined by reading the distance traveled by the positioning unit of the $3 \mathrm{D} \mathrm{LDV}$ in the $K_{L^{\prime}}$-frame. In reconstruction of the stapes motion without the misalignment considered, it was assumed that the orientation of the $K_{L^{\prime}}$-frame and $K_{0^{\prime}}$-frame coincided, which served as an error source. Thereby, the vector $\boldsymbol{r}_{O^{\prime} P_{i}}^{L^{\prime}}$, which is actually for a point $P_{i}$ in the $K_{L^{\prime}}$-frame, is assigned as the position vector $\boldsymbol{r}_{O^{\prime} P_{i}}$ of the measurement point $P_{i}$. Moreover, the displacements $u_{x i}$ and $u_{y i}$ measured with respect to the $K_{L^{\prime}}$-frame are interpreted as $\bar{u}_{x i}$ and $\bar{u}_{y i}$ in the $K_{0^{\prime}}$-frame. As a result, $u_{x i}$ and $u_{y i}$ of the point $P_{i}$ in the $K_{L^{\prime}}$-frame is interpreted as $\bar{u}_{x i}$ and $\bar{u}_{y i}$ of the point $\bar{P}_{i}$ in the $K_{0^{\prime}}$-frame.

To assess the consequences of the misalignment on the in-plane motion parameter, a physiological stapes motion at $1000 \mathrm{~Hz}$ and $94 \mathrm{~dB}$ SPL with three-degrees-of-freedom, without any in-plane motions in the $K_{0^{\prime}}$-plane, was also taken as an example. Then, Eqs. (A6) and (A7) deliver for a mean misalignment of $7.5^{\circ}$ with a standard deviation of $3.4^{\circ}$ the incorrect in-plane parameters: $\bar{T}_{x}=4.6-13.9 \%$, $\bar{T}_{z}=4.4-13.4 \%$, and $\bar{T}_{\beta}=0.7-1.8 \%$, although they are actually all zero.

\section{Conclusion of the error analysis}

The uncertainties that we have described resulted in unexpectedly high error boundaries of the determined inplane motions, particularly since the errors from the two sources (i.e., glass beads on the measurement surface and misalignment of the laser) could be superposed additively. Such errors influenced the search for the $K_{1}$-plane, which was hypothesized to exist within the footplate. Thus in searching for the $K_{1}$-plane, the uncertainties $b$ and the Bryant angles of the transformation matrix $S_{K_{0} K_{L^{\prime}}}$ from the $K_{0}$-frame to the $K_{L^{\prime}}$-frame were used as additional design variables in the minimization problem of Eq. (7). The hypothesis was confirmed that the $K_{1}$-plane is found to be within the footplate for values of $b$ and $S_{K_{0} K_{L^{\prime}}}$ within the mentioned uncertainty bounds.

For the extended minimization problem with additional consideration of error boundaries, the extended solution $\boldsymbol{p}^{*}=\left[x_{O O_{1}}^{*} y_{O O_{1}}^{*} z_{O O_{1}}^{*} \alpha_{K_{0} K_{1}}^{*} \beta_{K_{0} K_{1}}^{*} \gamma_{K_{0} K_{1}}^{*} b \alpha_{K_{0} L^{\prime}}^{*} \beta_{K_{0} L^{\prime}}^{*} \gamma_{K_{0} L^{\prime}}^{*}\right]$ was calculated such that it fulfilled the criterion in Eq. (7). The actual position vector $\boldsymbol{r}_{O P_{i}}$ of a measurement point $P_{i}$ and the displacements $\boldsymbol{u}_{K_{0^{\prime}}}$ at that point follow Eqs. (A1)-(A4) with the shift vector $\boldsymbol{r}_{O O^{\prime}}=[0 b 0]^{T}$. With $\boldsymbol{r}_{O P_{i}}$, the matrix $\boldsymbol{A}_{K 0^{\prime}}$ was built according to Eq. (2), and the vector $\boldsymbol{q}_{0}$ of the rigid-body motion components in the $K_{0}$-plane was calculated by Eq. (4). 


\section{RESULTS}

In Sec. III A, first the measured stapes motion in the $y_{0}$-direction, typically referred to as the piston-like motion, is compared to data reported in the literature. Next, the six-degrees-of-freedom motion of the stapes and the in-plane parameters are shown with respect to the actual measurement frames $K_{0^{\prime}}$ and $K_{L^{\prime}}$, respectively, and with respect to the identified $K_{1}$-frame. Finally, the position and orientation of the $K_{1}$-plane with respect to $K_{0}$ and the corresponding uncertainty parameters $b$ and $S_{K_{0} K_{L^{\prime}}}$ are given.

\section{A. Comparison with data in the literature}

Figure 7 illustrates the mean piston displacement of the stapes normalized by the ear canal pressure in comparison with the data of Asai et al. (1997), Hato et al. (2003), Sim et al. (2010a), and the ASTM standard F2505.24930 (displacement limits 5\%/95\% percentile interval) for acceptance as mechanically normal human TBs). As drained cochleae were used in this study, the measurement was expected to be different from the measurement with intact cochleae. Hato et al. (2003) showed that the drainage affects mainly the piston-like motion and only to a lesser extent the rocking-like motions and reduces the mass and damping significantly. That is why the first resonance gets more prominent, is shifted a little toward higher frequencies, and thus the displacement magnitude rises in the higher frequency range. However, the qualitative behavior of the transfer function stays the same concerning the low-frequency range and the roll-off of about $40 \mathrm{~dB} / \mathrm{decade}$. According to Hato et al. (2003), the rocking-like motions relative to the pistonlike motion are smaller with an intact cochlea than the corresponding values for the drained cochlea. From a classical theory of the viscous flow of Newtonian fluid, assuming the medial surface of the footplate as flat, shear stress caused by the in-plane motion of the stapes is presumed to be much

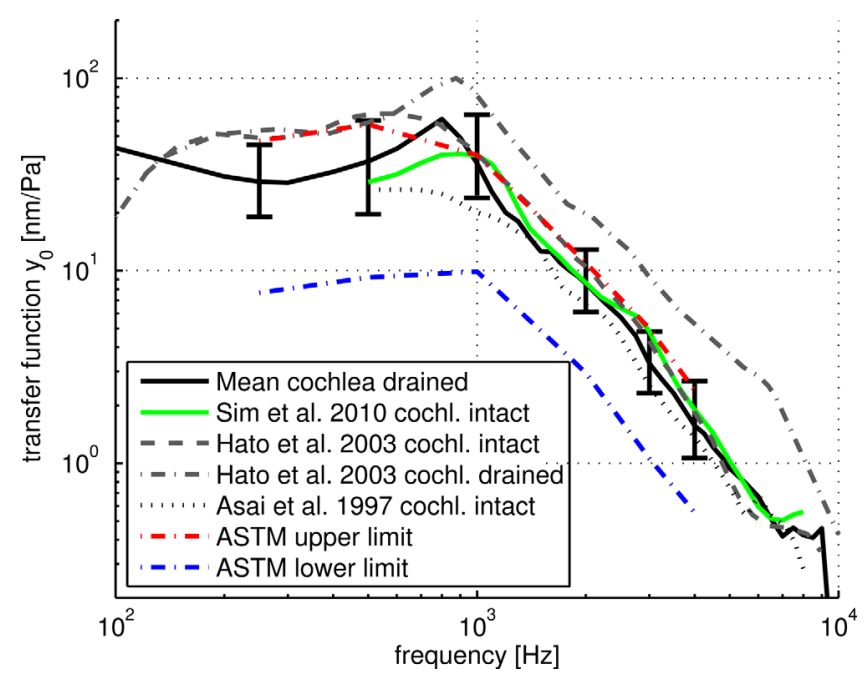

FIG. 7. (Color online) Measured mean $(n=5) y_{0}$-displacement (piston-like) of the stapes normalized by the sound pressure at the tympanic membrane in comparison with the data of Sim et al. (2010a), Hato et al. (2003), Asai et al. (1997), and the ASTM standard F2505.24930. The error bars represent the standard deviation. Measurements with intact and drained cochlea are differentiated. smaller than normal stress by the piston-like motion of the stapes. Therefore, we expect in the case of an intact inner ear a similar amount of in-plane motions or even slightly smaller in-plane motions than in the considered case of drained cochlea.

In our measurements, the first prominent resonance frequency was at around $850 \mathrm{~Hz}$ varying between $800 \mathrm{~Hz}$ and $1100 \mathrm{~Hz}$ across the five TBs. This corresponds to the findings of Silman and Silverman (1991) with the main resonance frequency at around 0.8 to $1.2 \mathrm{kHz}$ and to the findings of Wada et al. (1998) with a mean value of $1.17 \pm 0.27 \mathrm{kHz}$ based on 275 ears.

The measured mean piston-like displacement along with the corresponding standard deviation was within the ASTM limits, indicating that the five TBs used in our study were within a normal range. Since the ASTM standard relates to an intact cochlea, it seems reasonable that our measurements are at or slightly above the upper limit.

\section{B. Motions of the stapes with respect to the actual measurement frame ( $K_{L^{\prime}}$-frame)}

Figure 8 illustrates the mean magnitude of the three translational (A) and the three rotational (B) motions of the stapes calculated from the measurements on the medial surface of the stapes footplate in the five TBs. The magnitudes of the motion components were normalized by the sound pressure at the tympanic membrane and the corresponding standard deviations are indicated by shaded error corridors. The magnitudes were calculated as described in Sec. II C.

Motion of the medial-superior bony part outside the footplate ( $1 \mathrm{~mm}$ far from the annular ligament) was measured, and it was considered as a noise level including the structural vibration of the whole TB. In Fig. 8(A), the mean of all noise levels in all five TBs was plotted together with the translational motion components. Concerning the piston motion $y_{0^{\prime}}$, the mean signal-to-noise ratio (SNR) was larger than $40 \mathrm{~dB}$ across all frequencies and thus in a feasible range. Concerning the other translational components $x_{0^{\prime}}$ and $z_{0^{\prime}}$, whose magnitudes were much smaller, the SNR was feasible (larger than $20 \mathrm{~dB}$ ) for frequencies from 300 to $7000 \mathrm{~Hz}$ with an exception in the $z_{0^{\prime}}$ component around $4000 \mathrm{~Hz}$.

Figure 9 illustrates the reconstructed in-plane motion parameters of the $K_{O^{\prime}}$-plane, which was presumed to include errors of uncertainties from the glass beads and the laser misalignment. The corresponding standard deviations are illustrated with shaded error corridors. It can be seen that with some exceptions the in-plane motion parameters were nearly constant from 300 to $7000 \mathrm{~Hz}$.

Table I lists the mean values of the in-plane motion parameter across the frequency range from 300 to $7000 \mathrm{~Hz}$ for each TB. Moreover, the mean and the standard deviation across all five TBs were stated. It is striking that $x_{0^{\prime}}$ can assume very high values; at some frequencies it was $30 \%$ of the piston-like motion $\left(T_{x}=0.3\right)$.

The phases of the transfer functions are not shown in detail because our main focus is on the comparison between in-plane and out-of-plane magnitudes. Basically, the phase shift increased slowly below $1 \mathrm{kHz}$ and then became faster 

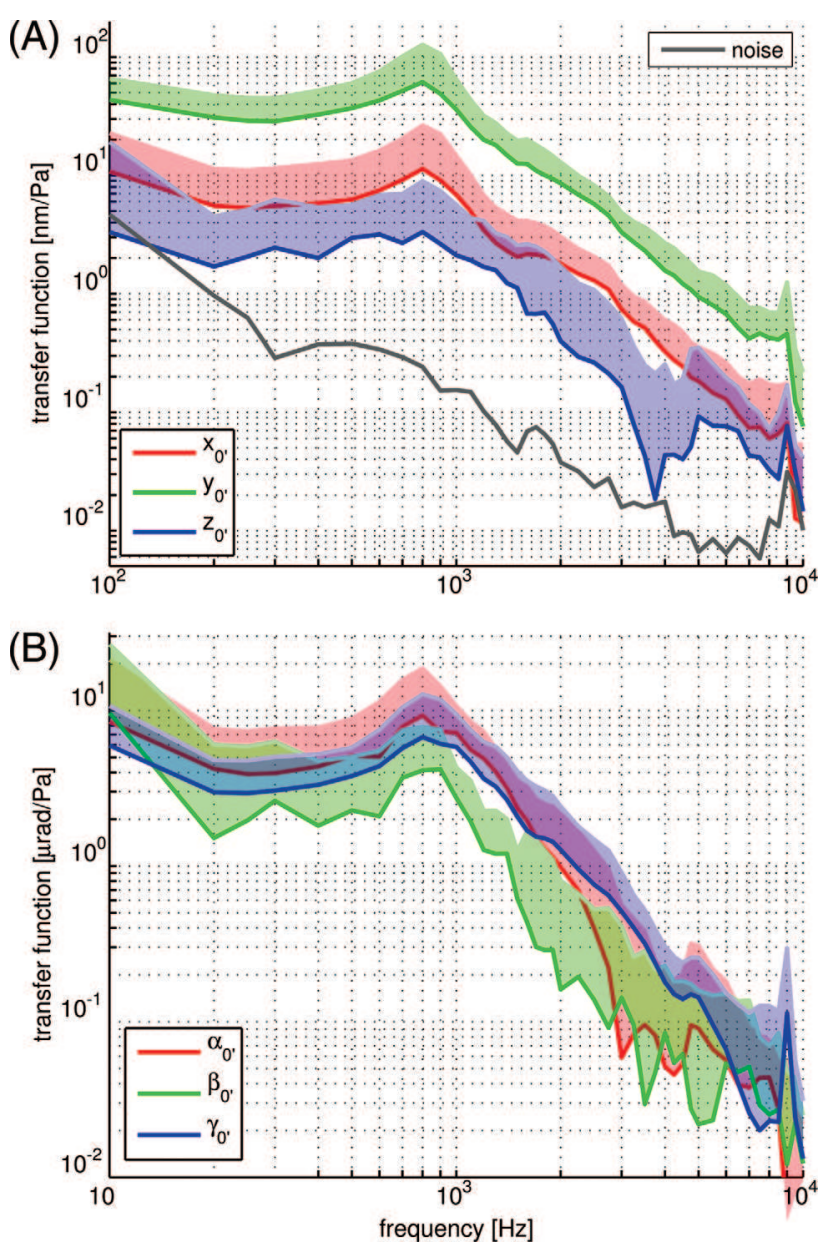

FIG. 8. Mean magnitude of the three translational (A) and the three rotational (B) stapes motion components, calculated from measurements in five TBs (see Sec. IIC) with respect to the actual measurement frame $K_{L^{\prime}}$. The magnitudes are normalized by the sound pressure at the tympanic membrane and the standard deviation is illustrated by shaded error corridors. The mean of the structural vibrations in all TBs, representing the noise level, is also displayed.

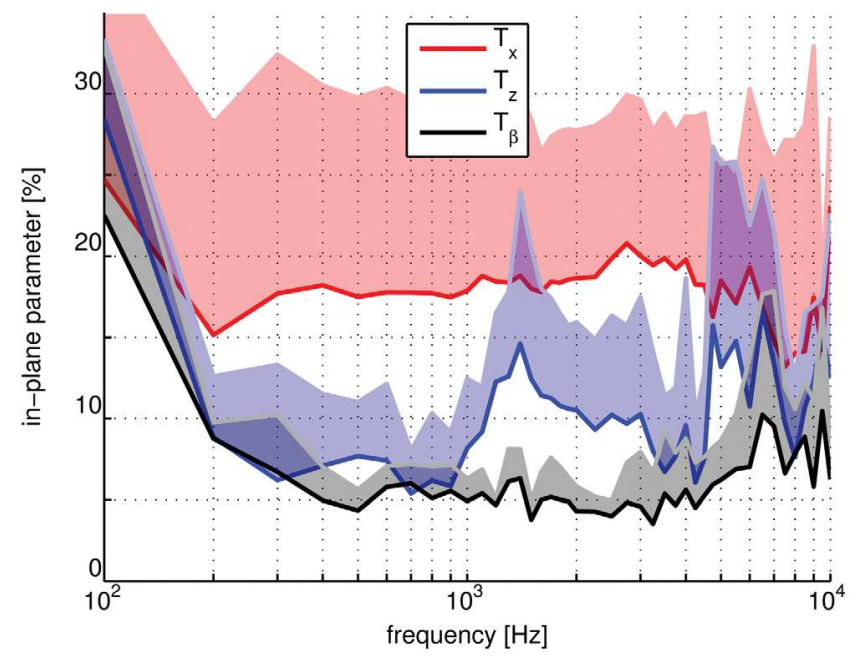

FIG. 9. (Color online) In-plane parameters of the $K_{0^{\prime}}$-plane including laser misalignment errors and corresponding standard deviations with shaded error corridors.
TABLE I. In-plane parameters of the $K_{0^{\prime}}$-plane including laser misalignment errors and of the $K_{1}$-plane and $K_{0}$-plane in all five TBs. The values are calculated for the frequency range from 300 to $7000 \mathrm{~Hz}$ in which a meaningful SNR is guaranteed.

\begin{tabular}{|c|c|c|c|c|c|c|c|c|c|}
\hline \multirow{2}{*}{$\begin{array}{l}\text { TB } \\
\text { No. }\end{array}$} & \multicolumn{3}{|c|}{$\begin{array}{c}\text { In-plane parameter } \\
\text { of } K_{0^{\prime}}[\%]\end{array}$} & \multicolumn{3}{|c|}{$\begin{array}{c}\text { In-plane parameter } \\
\text { of } K_{1}[\%]\end{array}$} & \multicolumn{3}{|c|}{$\begin{array}{c}\text { In-plane parameter } \\
\text { of } K_{0}[\%]\end{array}$} \\
\hline & $T_{x}$ & $T_{z}$ & $T_{\beta}$ & $T_{x}$ & $T_{z}$ & $T_{\beta}$ & $T_{x}$ & $T_{z}$ & $T_{\beta}$ \\
\hline 1 & 25.0 & 15.0 & 7.4 & 2.5 & 3.7 & 7.3 & 9.2 & 8.2 & 7.4 \\
\hline 2 & 22.4 & 15.9 & 6.7 & 3.6 & 8.6 & 5.7 & 9.2 & 9.0 & 6.1 \\
\hline 3 & 21.0 & 7.1 & 4.1 & 2.8 & 4.0 & 4.2 & 8.6 & 9.6 & 4.4 \\
\hline 4 & 3.8 & 7.1 & 4.1 & 2.2 & 3.3 & 3.3 & 5.2 & 7.3 & 4.3 \\
\hline 5 & 23.5 & 8.8 & 5.7 & 3.6 & 5.0 & 4.7 & 10.3 & 6.6 & 5.4 \\
\hline Mean & 19.1 & 10.8 & 5.6 & 2.9 & 4.9 & 5.0 & 8.5 & 8.1 & 5.5 \\
\hline Std & 8.7 & 4.3 & 2.5 & 0.6 & 2.2 & 1.5 & 3.2 & 3.6 & 2.4 \\
\hline
\end{tabular}

above leading to a phase shift of about 1.6 cycles around $10 \mathrm{kHz}$.

The reported spatial motion of the stapes at low frequencies was confirmed by these measurements. While the stapes head was moving inwards (medial direction), the motion direction of the stapes head was pointing along the superior, anterior direction. While the stapes head was moving outwards (lateral direction), the motion direction of the head was pointing along the inferior, posterior direction.

\section{Motions of the stapes with respect to the $K_{1}$-frame}

Figure 10 illustrates the measured stapes motion described in the $K_{1}$-frame. The three translations [Fig. 10(A)] and the three rotations [Fig. 10(B)] have been normalized by the ear canal pressure and are displayed together with the corresponding standard deviations. In order to track the changes from the representation in the actual measurement frames to the one in the $K_{1}$-frame, the mean magnitudes of the motion components described in the $K_{L^{\prime}}$-frame are displayed in gray. It can be seen clearly that the in-plane translations $x_{1}$ and $z_{1}$ were substantially lower, whereas the piston-like motion and all rotational motion components changed only slightly.

This finding is also mirrored by the in-plane motion parameters of the $K_{1}$-plane illustrated in Fig. 11. The in-plane parameters were reduced drastically, primarily in the frequency range of 300 to $7000 \mathrm{~Hz}$ where the SNR was high. The in-plane motion parameters did not change at frequencies outside the 300 to $7000 \mathrm{~Hz}$ range, where the SNR was low, and thus much measurement error was expected.

In Table I the mean in-plane motion parameters of the $K_{1}$-plane are listed in comparison to the corresponding values of the $K_{O^{\prime}}$-plane and the $K_{O}$-plane. The average across the frequency range between 300 and $7000 \mathrm{~Hz}$ is given for each of the five TBs. Moreover, the standard deviations among all five TBs are listed. $T_{x}$ is reduced drastically from $19.1 \%$ to $2.9 \%, T_{z}$ from $10.8 \%$ to $4.9 \%$, and $T_{\beta}$ from $5.6 \%$ to $5.0 \%$.

The in-plane motion parameters of the $K_{1}$-plane allow us to conclude that for the majority of measurements, the inplane motions in the $K_{1}$-plane were negligible compared to 

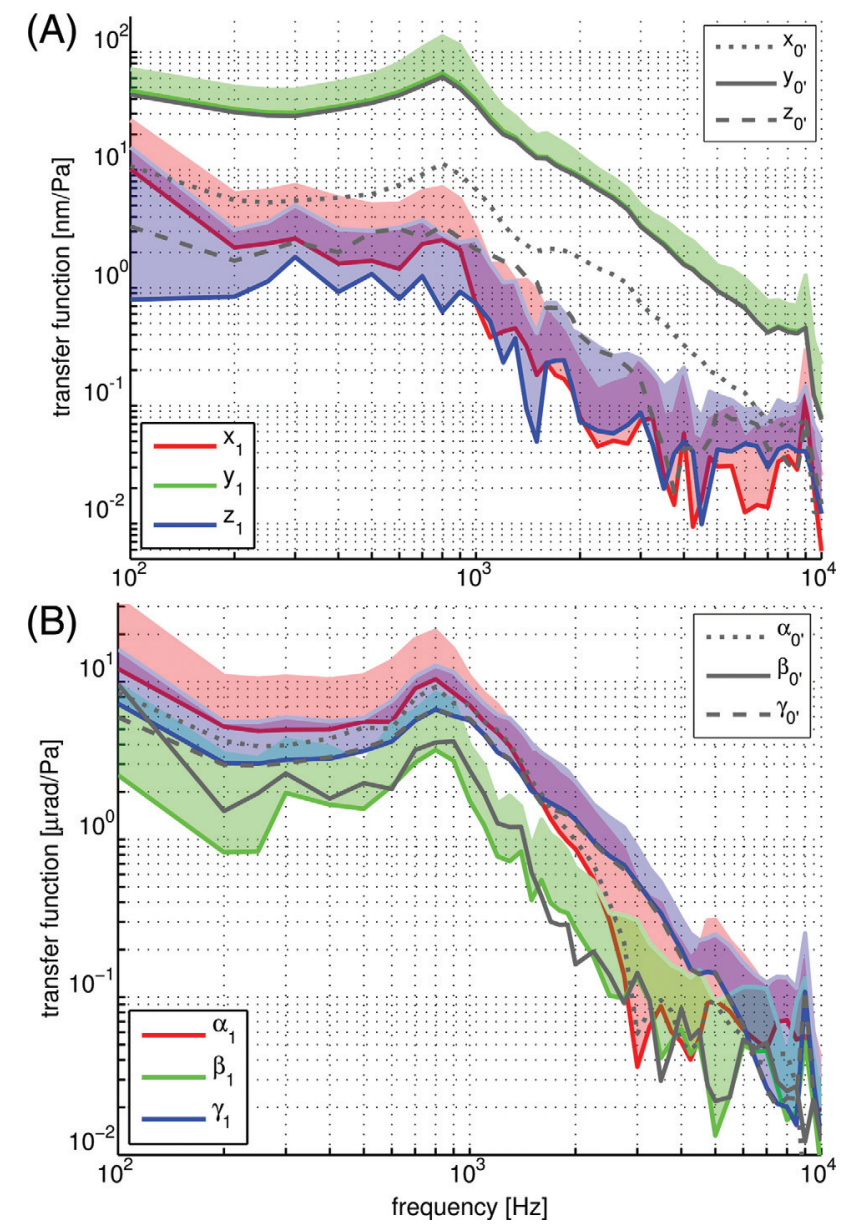

FIG. 10. Mean normalized magnitudes and the corresponding standard deviations of the three translational (A) and the three rotational (B) stapes motion components with respect to the stapes frame $K_{1}$. By comparison, the mean magnitudes of the stapes motions in $K_{L^{\prime}}$ (see Fig. 8) are displayed in gray.

the out-of-plane motions. The three in-plane motion parameters were around $4 \%$.

Table II provides the position and orientation of the $K_{1}$-frame with respect to the $K_{0}$-frame for all five TBs. In

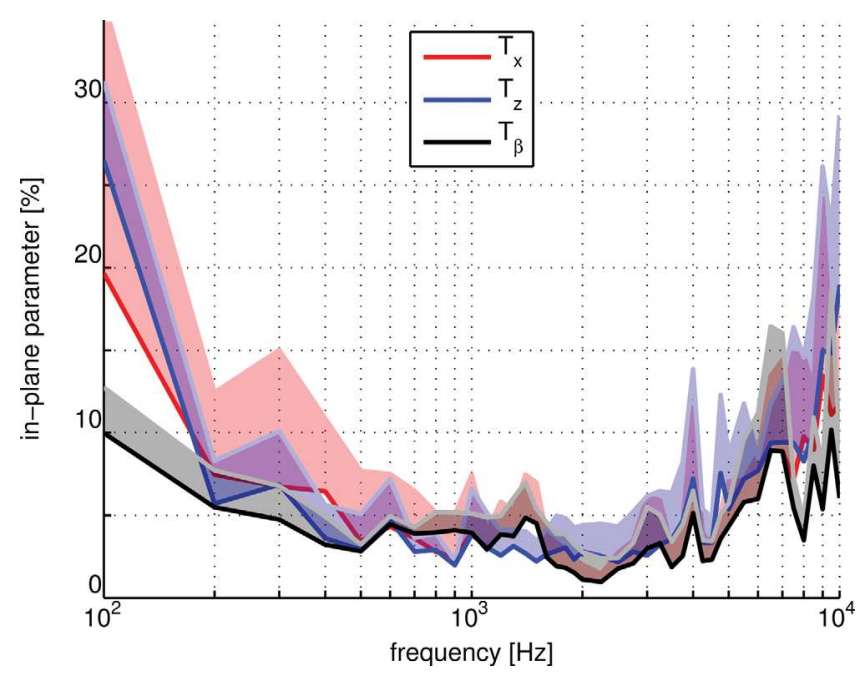

FIG. 11. (Color online) In-plane parameters related to the stapedial joint frame $K_{1}$ and corresponding standard deviations displayed with shaded error corridors.
TABLE II. Position and orientation of the $K_{1}$-frame with respect to the $K_{0}$-frame and the corresponding thickness $b$ of the glass beads layer and the laser orientation $S_{K_{0} K_{L^{\prime}}}$. The relative orientation between $K_{0^{-}}$and $K_{1}$-planes is given by the spatial angle $\psi$ and the relative orientation between $K_{0}$ - and $K_{L^{\prime}}$-planes by the angle $\varphi$. The shift of the origin is given by the coordinates of the shift vector $\boldsymbol{r}_{O O_{1}}$.

\begin{tabular}{|c|c|c|c|c|c|c|}
\hline \multirow{2}{*}{$\begin{array}{l}\text { TB } \\
\text { No. }\end{array}$} & \multicolumn{3}{|c|}{$\boldsymbol{r}_{O O_{1}}[\mu \mathrm{m}]$} & \multirow{2}{*}{$\begin{array}{c}\psi \\
{\left[{ }^{\circ}\right]}\end{array}$} & \multirow{2}{*}{$\begin{array}{c}b \\
{[\mu \mathrm{m}]}\end{array}$} & \multirow{2}{*}{$\begin{array}{c}\varphi \\
{\left[{ }^{\circ}\right]}\end{array}$} \\
\hline & $x$ & $y$ & $z$ & & & \\
\hline 1 & 259 & -196 & 241 & 3.2 & 341 & 6.4 \\
\hline 2 & 273 & -58 & 266 & 4.7 & 59 & 9.8 \\
\hline 3 & 298 & -167 & -268 & 5.7 & 252 & 7.4 \\
\hline 4 & 287 & -117 & 194 & 5.4 & 121 & 1.6 \\
\hline 5 & 289 & -51 & 297 & 4.5 & 51 & 7.1 \\
\hline Mean & 281 & -118 & 146 & 4.7 & 165 & 6.5 \\
\hline Std & 15.3 & -64 & 234.5 & 1.0 & 127 & 3 \\
\hline
\end{tabular}

Table II, an angle $\psi$ represents the spatial angle between the $y_{K_{0}}$-axis and the $y_{K_{1}}$-axis, and a vector $\boldsymbol{r}_{O O_{1}}$ represents the shift of the origin of the $K_{1}$-frame relative to the origin of the $K_{0}$-frame. Moreover, the thickness $b$ of the glass bead layer and the spatial angle $\varphi$, which is an angle between the $y_{K_{0^{\prime}}}$-axis and the $y_{K_{L^{\prime}}}$-axis, and was determined by the transformation matrix $\boldsymbol{S}_{K_{0} K_{L^{\prime}}}$, are also listed in Table II.

The mean of the $x$ and $z$ shifts in $\boldsymbol{r}_{O O_{1}}$ were both positive at around $281 \mu \mathrm{m}$ and $146 \mu \mathrm{m}$, respectively. The shifts in the $y_{K_{0}}$-direction were around $118 \mu \mathrm{m}$ pointing laterally. The mean angle $\psi$ between the $K_{0}$-plane and the $K_{1}$-plane was $4.7^{\circ}$.

In all cases except one, $b$ was within the estimated bounds of about 50 to $300 \mu \mathrm{m}$. The minimum value of $51 \mu \mathrm{m}$ was the approximate thickness of a single glass bead layer. The values of the angle $\varphi$ were also feasible when taking into account the uncertainty bounds for the laser misalignment, which were around $7.5^{\circ}$ with a standard deviation of $3.4^{\circ}$.

\section{DISCUSSION}

\section{A. Anatomical feasibility of the $K_{1}$-plane}

The decomposition of motions into in-plane and out-ofplane components depends on the position and orientation of the plane under consideration. The results of this study have shown that the in-plane motions were large for the measurement plane (i.e., $K_{L^{\prime}}$-plane) but they became much smaller (in-plane parameter around 4\%) for the slightly shifted and rotated $K_{1}$-plane. Considering the hypothesis of this study that the in-plane motions become minimal in a certain crosssectional plane of the stapes footplate, the question is: Can the determined positions and orientations of the $K_{1}$-planes be anatomically feasible?

Concerning the position of the $K_{1}$-plane, the $x$ and $z$ shifts of the origin of $0.28 \mathrm{~mm}$ in the $x$-direction and 0.15 $\mathrm{mm}$ in the $z$-direction were less than $10 \%$ considering the lengths of the footplate along its long axis (approximately $3 \mathrm{~mm}$ ) and along its short axis (approximately $1.5 \mathrm{~mm}$ ) (see Kirikae, 1960; Farahani and Nooranipour, 2008). Furthermore, the $K_{1}$-plane was shifted to the lateral direction by 
$118 \mu \mathrm{m}$ on average, relative to the $K_{0}$-plane (c.f. Table II). Taking into account a footplate thickness of about $250 \mu \mathrm{m}$ as reported by Farahani and Nooranipour (2008), the $K_{1}$-plane is located near the middle of the stapes footplate.

Anatomical feasibility of the rotation of the $K_{1}$-plane relative to the $K_{0}$-plane $\left(4.7 \pm 1^{\circ}\right)$ was examined with the $3 \mathrm{D}$ feature of the stapes footplate reconstructed from micro-CT images. Figure 12 displays the 3D feature of a male stapes reconstructed from micro-CT images. The $K_{0}$-plane (red plane) was determined as a plane fitting to the medial surface of the footplate with the centroid of the medial surface as origin. The vertical axis of the stapes defined as a line connecting the origin of $K_{0}$ and the stapes head, deviated by $7.5 \pm 1.3^{\circ}$ from the normal $\left(y_{K_{0}}\right.$-axis) of the $K_{0}$-plane in the five TBs. Hence, the inclination of the $K_{1}$-plane relative to the $K_{0}$-plane by $4.7 \pm 1^{\circ}$ is feasible considering the anatomy of the stapes footplate.

We can thus conclude that spatial motion of the stapes exhibits negligible in-plane components in the $K_{1}$-plane, which is located near the middle of the footplate with a mean distance of $118 \mu \mathrm{m}$ from the $K_{0}$-plane and an inclination of $4.7^{\circ}$ relative to the $K_{0}$-plane.

\section{B. Reconstructed in-plane parameters in the $K_{0}$-plane}

In Sec. III, the in-plane motions on the $K_{1}$-plane were shown in comparison to the in-plane motions on the measurement plane (i.e., $K_{L^{\prime}}$-plane). Based on these findings, the question of how large the in-plane motions are in the $K_{0}$-plane can be raised. The in-plane motions on the $K_{0}$-plane were calculated with mean relative position between the $K_{0}$-plane and the $K_{1}$-plane and the stapes motion in the $K_{1}$-frame, which corresponds to Table II. Equations (5) and (6) were used for the calculation.

Figure 13 illustrates the resulting in-plane motion parameters for the $K_{0}$-plane, which are listed as well in Table $\mathrm{I}$ in detail. $T_{x}$ and $T_{z}$ were about $8.5 \%$ and $8.1 \%$, respectively, and $T_{\beta}$ was about $5.5 \%$ in the frequency range from 300 to $7000 \mathrm{~Hz}$. While $T_{\beta}$ showed similar values for

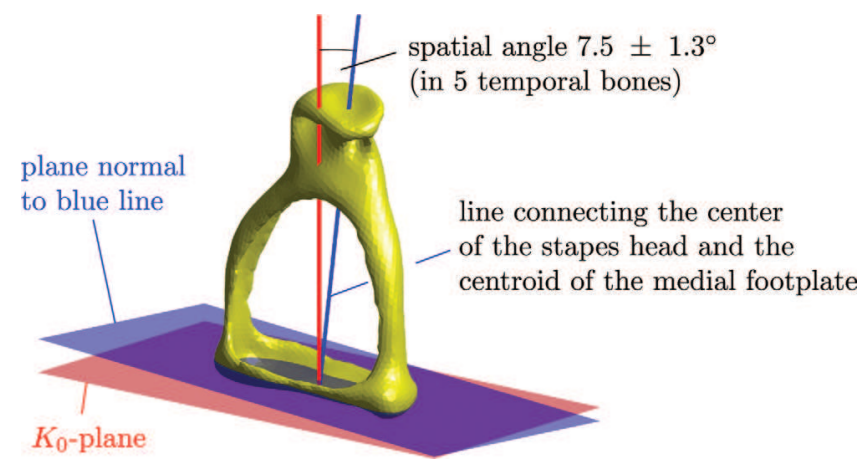

FIG. 12. Red represents a plane fitting to the lateral surface of the footplate surface with the centroid of the surface as the origin $\left(K_{0}\right.$-plane). Blue depicts the shift of the plane to the lateral direction by $118 \mu \mathrm{m}$ relative to the $K_{0^{-}}$ plane and was rotated such that it became normal to a line connecting the centroid of the medial footplate surface and the centroid of the stapes head (blue line).

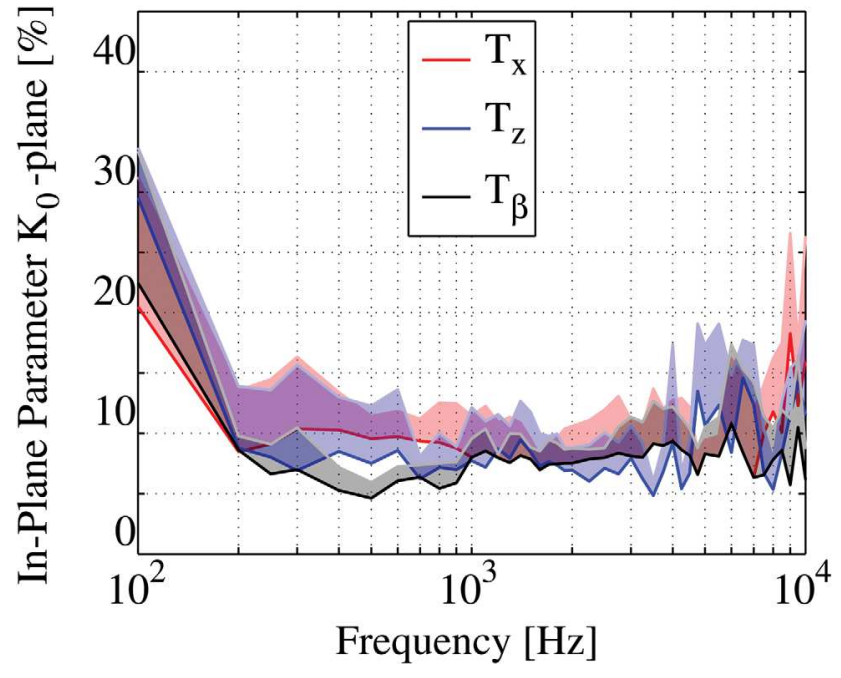

FIG. 13. (Color online) Reconstructed in-plane parameters of the $K_{0}$-plane (or lateral footplate plane) based on the stapes motion in the $K_{1}$-plane. The $K_{1}$-plane was shifted $118 \mu \mathrm{m}$ in the lateral direction and rotated by $4.7^{\circ}$ with respect to the $K_{0}$-plane.

$K_{0^{\prime}}$ and $K_{1}$-planes, $T_{x}$ and $T_{z}$ on the $K_{0^{\prime}}$-plane were larger than the corresponding values on the $K_{1}$-plane.

\section{Consequences of in-plane motions on reconstruction of stapes motions from measurements using 1D and scanning LDV}

As in-plane motions are normally present in the $K_{0}$-plane as shown in Fig. 13, they will affect reconstructed stapes motions when determined by measurements using a one-dimensional (1D) or SLDV system, where reduced degrees of freedom of the stapes motion are usually assumed. As the 1D LDV or SLDV measures motion of the target only along the laser beam direction, the full six-degrees-of-freedom motion of the stapes cannot be reconstructed if motions of points on the medial or lateral surface of the footplate are measured without changing relative angles between the footplate and the laser beam of the LDV. In previous works by Hato et al. (2003) and by Sim et al. (2010a, 2010b), pistonand rocking-like motions of the stapes footplate were reconstructed from their limited measurements, assuming no in-plane motion of the footplate. However, the reconstructed piston- and rocking-like motions contain a certain amount of error due to the in-plane motions, which were ignored in their reconstructions.

Figure 14 depicts two measurement situations. With no in-plane motion, depicted on the left side, $u_{i}$ equals $u_{y_{i}}$ and the LDV measures $u_{m}=u_{i} \cos (\varphi)$. When in-plane motions are present, depicted on the right side, the displacement vector $u_{i}$ at a measurement point on the footplate contains the $x$ and $z$ components $u_{x_{i}}$ and $u_{z_{i}}$ as well as the component $u_{y_{i}}$. As illustrated in Fig. 14, this yields $u_{m}=\cos (\varphi)\left(u_{x_{i}}+u_{z_{i}}\right)$. When measurements are made with the 1D LDV system, the spatial motion components cannot be measured separately, and it is generally assumed that $u_{x_{i}}$ and $u_{z_{i}}$ equals zero and thus $u_{i}=u_{y_{i}}=u_{m} / \cos (\varphi)$. As Fig. 14 illustrates, this is obviously not true and leads to an inaccurate reconstruction of the piston motion $y_{0}$ and rocking motions $\alpha_{0}$ and $\gamma_{0}$. 
without in-plane motions
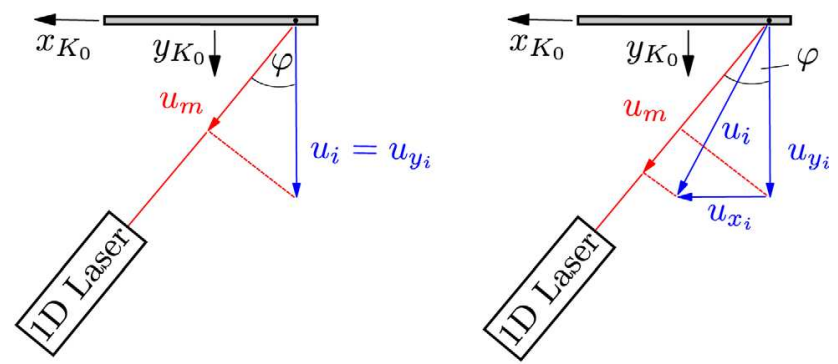

FIG. 14. (Color online) Consequence of in-plane motions on reduced 1D/SLDV measurements. Except for the case of no in-plane motions, the assumption $u_{i}=u_{y_{i}}=u_{m} / \cos (\varphi)$ introduces an error in the reconstructed piston and rocking motions of the stapes, because the measured displacement $u_{m}$ generally consists of all three spatial components $u_{x_{i}}, u_{y_{i}}$, and $u_{z_{i}}$.

In order to estimate the induced error, the stapes motion of Fig. 13 was prescribed in the $K_{0}$-frame and a 1D SLDV measurement on the medial surface was simulated. In the simulation, it was assumed that $K_{L^{\prime}}=K_{0^{\prime}}=K_{0}$. While the measured motion $u_{m}$ along the direction of the laser beam was actually simulated from $u_{x_{i}}, u_{y_{i}}$, and $u_{z_{i}}$, reconstruction of $u_{y_{i}}$ was done by $u_{y_{i}}=u_{m} / \cos (\varphi)$ with the assumption of the reduced degrees of freedom (i.e., $u_{x_{i}}=u_{z_{i}}=0$ ). Such discrepancy served as an error source on the reconstructed $u_{y_{i}}$ and thus reconstructed rigid body motions.

The errors in $y_{0}, \alpha_{0}$, and $\gamma_{0}$ were qualitatively and quantitatively very similar. With $0^{\circ}$ inclination of the laser beam (i.e., the laser beam is perpendicular to the $K_{0}$-plane), no error was induced to $y_{0}, \alpha_{0}$, and $\gamma_{0}$ by the in-plane motions because in-plane motions do not affect the measured $u_{y_{i}}$-component of the point. With an inclination angle of $45^{\circ}$, the error in the piston-like displacement $y_{0}$ was $3.7 \mathrm{~dB}$, the error in $\alpha_{0}$ was $3.1 \mathrm{~dB}$, and the error in $\gamma_{0}$ was $2.8 \mathrm{~dB}$. Considering typical inclinations of less than $45^{\circ}$, it can be concluded that the effect of in-plane motions on the reconstructed outof-plane motion components, which are generally measured with 1D LDV or SLDV systems, is small.

\section{CONCLUSIONS}

In this study, the six-degrees-of-freedom stapes motions were reconstructed from measurements on the medial surface of the stapes footplate, and in-plane motions of the footplate were investigated for the measurement plane ( $K_{L^{\prime}}$-plane), the approximated plane of the medial footplate surface ( $K_{0}$-plane), and the cross-sectional plane of the footplate in which the in-plane motions became minimal $\left(K_{1}\right.$-plane). For illustration of the in-plane motions, in-plane motion parameters were defined as the ratio of the in-plane motions (two translational and one rotational) to the translational out-of-plane motion. We determined the existence of the $K_{1}$-plane, which has in-plane motion parameters of less than $5.0 \%$. The position and orientation of the $K_{1}$-plane were in an anatomically feasible range. Simulated experiments were carried out to see the effects of the in-plane motions on reconstructions of stapes motions using $1 D$ LDV or SLDV systems. With typical inclinations of the laser relative to the stapes footplate, errors on the reconstructed out-of-plane motions caused by the in-plane motions are expected to be less than $4.0 \mathrm{~dB}$.

\section{ACKNOWLEDGMENT}

This work was supported by Deutsche Forschungsgemeinschaft EI-231-4/2 Grants.

\section{APPENDIX: EFFECTS OF THE UNCERTAINTIES IN THE MEASUREMENT POSITION AND IN THE ALIGNMENT OF THE LASER ON THE IN-PLANE PARAMETER}

Uncertainties in the measurement position and in the alignment of the 3D LDV affect the determined in-plane parameter. Figure 15 depicts three frames of reference introduced in this article: A frame based on the medial surface of the footplate ( $K_{0}$-frame), a frame shifted from the $K_{0}$-frame due to glass beads ( $K_{0^{\prime}}$-frame), and a frame determined by the laser beam of the $\operatorname{LDV}\left(K_{L^{\prime}}\right.$-frame). The $K_{0}$-frame can be considered as an ideal measurement frame without any offset and alignment error of the laser beam.

As the shift from the $K_{0}$-frame to the $K_{0^{\prime}}$-frame is defined by the vector $\boldsymbol{r}_{O O^{\prime}}$, the position vector $\boldsymbol{r}_{O P_{i}}$ of a measurement point $P_{i}$ in the $K_{0}$-frame is expressed as the corresponding position vector $\boldsymbol{r}_{O^{\prime} P_{i}}$ in the $K_{0^{\prime}}$-frame.

$$
\boldsymbol{r}_{O P_{i}}=\boldsymbol{r}_{O O^{\prime}}+\boldsymbol{r}_{O^{\prime} P_{i}} .
$$

Then the corresponding position vector $\boldsymbol{r}_{O^{\prime} P_{i}}^{L^{\prime}}$ represented in coordinates of the $K_{L^{\prime}}$-frame is related to the position vector $r_{O^{\prime} P_{i}}$ in the $K_{0^{\prime}}$-frame as

$$
\boldsymbol{r}_{O^{\prime} P_{i}}^{L^{\prime}}=\boldsymbol{S}_{K_{0} K_{L^{\prime}}} \boldsymbol{r}_{O^{\prime} P_{i}}
$$

where $S_{K_{0} K_{L^{\prime}}}$ represents a rotational transformation matrix from the $K_{0^{\prime}}$ frame to the $K_{L^{\prime}}$ frame. With the $y$-component of $\boldsymbol{r}_{O^{\prime} P_{i}}$ as zero, Eqs. (A1) and (A2) result in

$$
\boldsymbol{r}_{O^{\prime} P_{i}}^{L^{\prime}}=\left\{\begin{array}{l}
S_{11}\left(x_{i}^{\prime}-x_{\text {shift }}\right)+S_{13}\left(z_{i}^{\prime}-z_{\text {shift }}\right) \\
S_{21}\left(x_{i}^{\prime}-x_{\text {shift }}\right)+S_{23}\left(z_{i}^{\prime}-z_{\text {shift }}\right) \\
S_{31}\left(x_{i}^{\prime}-x_{\text {shift }}\right)+S_{33}\left(z_{i}^{\prime}-z_{\text {shift }}\right)
\end{array}\right\},
$$

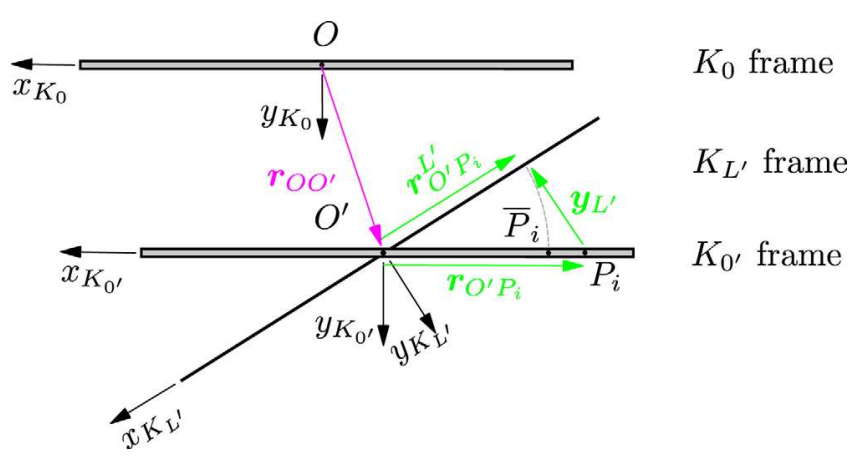

FIG. 15. (Color online) Due to uncertainties in the alignment of the stapes and the $3 \mathrm{D} \mathrm{LDV}$, the assumed frame $K_{0}$ and the actual frame $K_{L^{\prime}}$ and $K_{0^{\prime}}$ do not coincide. Consequently, there are errors in the positions of the measurement points and in the measured displacements at those points. 
with $\boldsymbol{r}_{O P_{i}}=\left[x_{i}^{\prime} y_{i}^{\prime} z_{i}^{\prime}\right]^{T}$ and $\boldsymbol{r}_{O O^{\prime}}=\left[x_{\text {shift }} y_{\text {shift }} z_{\text {shift }}\right]^{T}$. Here, $S_{i j}$ indicates an element of the matrix $S_{K_{0} K_{L^{\prime}}}$ in the $i$ th row and the $j$ th column. Without the offset by the glass beads and misalignment of the laser beam considered, $x$ and $z$ values of $\boldsymbol{r}_{O^{\prime} P_{i}}^{L^{\prime}}$ are wrongly assigned to $x$ and $z$ coordinates of the measurement point $P_{i}$ in the $K_{0}$-frame. Denoting the wrong position vector of the measurement point $P_{i}$ in the $K_{0}$-frame as $\overline{\boldsymbol{r}}_{O P_{i}}$

$$
\overline{\boldsymbol{r}}_{O P_{i}}=\left\{\begin{array}{c}
S_{11}\left(x^{\prime}{ }_{i}-x_{\text {shift }}\right)+S_{13}\left(z^{\prime}{ }_{i}-z_{\text {shift }}\right) \\
0 \\
S_{31}\left(x_{i}^{\prime}-x_{\text {shift }}\right)+S_{33}\left(z^{\prime}{ }_{i}-z_{\text {shift }}\right)
\end{array}\right\} .
$$

Correspondingly, the incorrect matrix $\overline{\boldsymbol{A}}_{K_{0}}$ is constructed from the wrong position vector $\overline{\boldsymbol{r}}_{O P_{i}}$.

Without consideration of the misalignment of the laser beam with respect to the $K_{0}$-frame, the measured displacement $\boldsymbol{u}_{K_{L^{\prime}}}$ in the $K_{L^{\prime}}$-frame is also incorrectly assigned to the corresponding displacement $\boldsymbol{u}_{i}$ in the $K_{0}$-frame. Therefore, the incorrect displacement vector $\overline{\boldsymbol{u}}_{i}$ in the $K_{0}$-frame becomes

$$
\overline{\boldsymbol{u}}_{i}=\left\{\begin{array}{l}
S_{11} u_{x_{i}}+S_{12} u_{y_{i}}+S_{13} u_{z_{i}} \\
S_{21} u_{x_{i}}+S_{22} u_{y_{i}}+S_{23} u_{z_{i}} \\
S_{31} u_{x_{i}}+S_{32} u_{y_{i}}+S_{33} u_{z_{i}}
\end{array}\right\}
$$

Consequently, by substituting the incorrect positions and displacements of the measurement points in the $K_{0}$-frame to Eq. (4), an incorrectly estimated rigid body motion $\overline{\boldsymbol{q}}_{0}$ in the $K_{0}$-frame is obtained as

$$
\overline{\boldsymbol{q}}_{0}=\left(\overline{\boldsymbol{A}}_{K_{0}}^{T} \overline{\boldsymbol{A}}_{K_{0}}\right)^{-1} \overline{\boldsymbol{A}}_{K_{0}}^{T} \overline{\boldsymbol{u}}_{K_{0}},
$$

where $\overline{\boldsymbol{u}}_{K_{0}}=\left[\overline{\boldsymbol{u}}_{1} \overline{\boldsymbol{u}}_{2} \cdots \overline{\boldsymbol{u}}_{n}\right]^{T}$ for nine measurement points $(n=9)$. With $\overline{\boldsymbol{u}}_{0}=\left[\bar{x}_{0} \bar{y}_{0} \bar{z}_{0} \bar{\alpha}_{0} \bar{\beta}_{0} \bar{\gamma}_{0}\right]^{T}$, the incorrect in-plane motion parameters are obtained as

$$
\bar{T}_{x}=\frac{\left|\bar{x}_{0}\right|}{\left|\bar{y}_{0}\right|}, \quad \bar{T}_{z}=\frac{\left|\bar{z}_{0}\right|}{\left|\bar{y}_{0}\right|} \text { and } \bar{T}_{\beta}=\frac{\left|\bar{\beta}_{0}\right| 0.7 \mathrm{~mm}}{\left|\bar{y}_{0}\right|} .
$$

Asai, M., Roberson, J., and Goode, R. L. (1997). "Acoustic effect of malleus head removal and tensor tympani muscle section on middle ear reconstruction," Laryngoscope 107, 1217-1222.
Chien, W., Ravicz, M., Merchant, S., and Rosowski, J. (2006). "The effect of methodological differences in the measurement of stapes motion in live and cadaver ears," Audiol. Neuro-Otol. 11, 183-197.

Decraemer, W., and Khanna, S. (1999). "New insights in the functioning of the middle ear," in The Function and Mechanics of Normal, Diseased and Reconstructed Middle Ears, edited by J. J. Rosowski and S. N. Merchant (Kugler Publications, The Hague, Netherlands), pp. 23-38.

Decraemer, W. F., de La Rochefoucauld, O., Dong, W., Khanna, S. M., Dirckx, J. J. J., and Olson, E. S. (2007). "Scala vestibuli pressure and three-dimensional stapes velocity measured in direct succession in gerbil," J. Acoust. Soc. Am. 121, 2774-2791.

Farahani, R. M., and Nooranipour, M. (2008). “Anatomy and anthropometry of human stapes," Am. J. Otol. 29, 42-47.

Gundersen, T. (1972). "Prostheses in the ossicular chain," Arch. Otol. Clin. North Am. 96, 416-423.

Gyo, K., Aritomo, H., and Goode, R. L. (1987). "Measurement of the ossicular vibration ratio in human temporal bones by use of a video measuring system," Acta Oto-Laryngol. 103, 87-95.

Hato, N., Stenfelt, S., and Goode, R. L. (2003). "Three-dimensional stapes footplate motion in human temporal bones," Audiol. Neuro-Otol. 8, $140-152$.

Kirikae, J. (1960). The Middle Ear (University of Tokyo Press, Tokyo, Japan), pp. 109-110.

Ravicz, M. E., Cooper, N. P., and Rosowski, J. J. (2008). "Gerbil middle-ear sound transmission from $100 \mathrm{~Hz}$ to $60 \mathrm{kHz}$," J. Acoust. Soc. Am. 124, $363-380$.

Rosowski, J. J., Songer, J. E., Nakajima, H. H., Brinsko, K. M., and Merchant, S. N. (2004). "Clinical, experimental, and theoretical investigations of the effect of superior semicircular canal dehiscence on hearing mechanisms," Otol. Neurotol. 25, 323-332.

Ruggero, M. A. and Temchin, A. (2003). "Middle-ear transmission in humans: Wideband, not frequency-tuned?," ARLO 4, 53-58.

Silman, S., and Silverman, C. A. (1991). Auditory Diagnosis: Principles and Applications (Academic, San Diego), Chap. 3, p. 79.

Sim, J. H., Chatzimichailis, M., Lauxmann, M., Röösli, C., Eiber, A., and Huber, A. (2010a). "Complex stapes motion in human ears," J. Assoc. Res. Otolaryngol. 11, 329-341.

Sim, J. H., Chatzimichailis, M., Röösli, C., Laske, R., and Huber, A. (2012). "Objective assessment of stapedotomy surgery from round window motion measurement," Ear Hear. 33(5), e24-e31.

Sim, J. H., Lauxmann, M., Chatzimichailis, M., Röösli, C., Eiber, A., and Huber, A. (2010b). "Errors in measurement of three-dimensional motions of the stapes using a laser Doppler vibrometer system," Hear. Res. 270, 4-14.

von Békésy, G. (1960). Experiments in Hearing (McGraw-Hill Book Company, New York), pp. 95-104.

Voss, S. E., Rosowski, J. J., Merchant, S. N., and Peake, W. T. (2000). “Acoustic responses of the human middle ear," Hear. Res. 150, 43-69.

Wada, H., Koike, T., and Kobayashi, T. (1998). "Clinical applicability of the sweep frequency measuring apparatus for diagnosis of middle ear diseases," Ear Hear. 19, 240-249. 